\title{
Hypoglycemia following the use of glucagon-like peptide-1 receptor agonists: a real-world analysis of post-marketing surveillance data
}

\author{
Zhe Zhao ${ }^{1,2}$, Yan Tang ${ }^{1}$, Yang $\mathrm{Hu}^{1}$, Huijuan $\mathrm{Zhu}^{3}$, Xiaoguang $\mathrm{Chen}^{2}$, Bin Zhao ${ }^{1}$ \\ ${ }^{1}$ Department of Pharmacy, Peking Union Medical College Hospital, Chinese Academy of Medical Sciences \& Peking Union Medical College, \\ Beijing, China; ${ }^{2}$ State Key Laboratory of Bioactive Substrate and Function of Natural Medicine, Institute of Materia Medica, Chinese Academy \\ of Medical Sciences \& Peking Union Medical College, Beijing, China; ${ }^{3}$ Department of Endocrinology, Key Laboratory of Endocrinology of the \\ National Health Commission, Peking Union Medical College Hospital, Chinese Academy of Medical Sciences \& Peking Union Medical College, \\ Beijing, China \\ Contributions: (I) Conception and design: B Zhao; (II) Administrative support: H Zhu, X Chen, B Zhao; (III) Provision of study materials or patients: \\ Y Hu, Y Tang; (IV) Collection and assembly of data: Z Zhao; (V) Data analysis and interpretation: Z Zhao; (VI) Manuscript writing: All authors; (VII) \\ Final approval of manuscript: All authors. \\ Correspondence to: Dr. Bin Zhao. Department of Pharmacy, Peking Union Medical College Hospital, Chinese Academy of Medical Sciences \& Peking \\ Union Medical College, Beijing 100730, China. Email: zhaobin@pumch.cn.
}

Background: Recent evidence has emerged concerning hypoglycemia following the application of glucagon-like peptide-1 receptor agonists (GLP-1RAs). Nevertheless, few real-world investigations have been performed to determine the clinical characteristics, onset, and outcomes of hypoglycemia associated with different GLP-1RAs. This study aimed to compare and assess the relationship between various GLP1RAs and hypoglycemia in a large population based on updated data from the Food and Drug Administration Adverse Event Reporting System (FAERS).

Methods: Bayesian and disproportionality analyses were applied to data mining in order to investigate suspected cases of hypoglycemia following various GLP-1RAs using the FAERS data between January 2004 and September 2020. We also evaluated the onset time, fatality risks, and hospitalization proportions of GLP-1RA-related hypoglycemia.

Results: We identified 1,164 GLP-1RA-associated hypoglycemia cases, which seemed to affect more middle-aged patients than elderly ones. Also, females were more affected than males. Lixisenatide demonstrated a stronger association with hypoglycemia compared to other GLP-1RAs, according to the highest reporting odds ratio $(\mathrm{ROR})(28.03,95 \%$ confidence interval $=15.92,49.32)$, empirical Bayes geometric mean [26.00, 95\% confidence interval (CI): 16.20], and proportional reporting ratio (PRR) (26.01, $\chi^{2}=313.37$ ). The median time to hypoglycemia onset was 5 days (interquartile range, $0-67.75$ days) following GLP-1RA treatment. In general, GLP-1RA-associated hypoglycemia resulted in fatality and hospitalization proportions of $3.53 \%$ and $56.08 \%$, respectively.

Conclusions: By analyzing the FAERS data, we outlined the association between hypoglycemia and different GLP-1RAs in greater detail in terms of clinical features, onset, and outcomes. Among all six GLP1RAs, lixisenatide demonstrated the strongest association with hypoglycemia while no relationship between albiglutide and hypoglycemia was observed. Attention should be given to GLP-1RAs when used in patients with high risks of hypoglycemia.

Keywords: Glucagon-like peptide-1 receptor agonist (GLP-1RA); adverse event reporting system; hypoglycemia

Submitted Jul 26, 2021. Accepted for publication Sep 16, 2021.

doi: 10.21037/atm-21-4162

View this article at: https://dx.doi.org/10.21037/atm-21-4162 


\section{Introduction}

Glucagon-like peptide-1 (GLP-1) is a gut-derived peptide produced by intestinal cells after ingestion of glucose, exerting glucose-lowering effects by augmenting insulin secretion through activating GLP-1 receptor (1). Hence, a class of peptides with similar structures to GLP-1 which can also stimulate GLP-1 receptor, known as glucagon-like peptide-1 receptor agonists (GLP-1RAs), were developed to improve glucose control for treatment of type 2 diabetes (2). These drugs can be classified into two groups, long-acting (dulaglutide, albiglutide, semaglutide, liraglutide, and once-weekly exenatide) and short-acting (lixisenatide and twice-daily exenatide) regimens, based on the duration of action (1). To date, a total of six medications including liraglutide, exenatide, dulaglutide, albiglutide, lixisenatide, and semaglutide have been approved by the US Food and Drug Administration (FDA) for use in the management of type 2 diabetes (3). In addition to the outstanding glucoselowering effects on patients with type 2 diabetes, GLP-1RAs have also shown benefits in several cardiovascular outcome trials, including LEADER (4), SUSTAIN 6 (5), Harmony Outcomes (6), and REWIND (7). Therefore, in their 2019 guidelines, the European Association for the study of Diabetes (EASD) and the American Diabetes Association (ADA) recommend the addition of one GLP-1RA when treating patients at high risks of adverse cardiovascular events, in order to prevent related death $(8,9)$.

Despite their beneficial effects, some adverse events are reportedly associated with GLP-1RAs (10). The most frequently occurring events are gastrointestinal disorders, such as nausea, diarrhea, vomiting, abdominal pain, and so on (11). Also, it is concerning that the use of GLP-1RAs may be related to an elevated risk of acute pancreatitis, and the FDA has warned the public about this adverse event associated with exenatide usage (12). Although GLP-1RAs are often thought to be linked to a relatively lower risk of hypoglycemia (13), the combination of GLP-1RAs and other anti-diabetic medications (e.g., sulphonylureas) have shown an elevated risk of hypoglycemia compared to placebo (14). In one clinical trial, the incidence of hypoglycemia $(9.2 \%)$ in the group treated with liraglutide $(1.2 \mathrm{mg} /$ day $)$ combined with glimepiride was significantly increased compared to the placebo group (2.6\%) (15). Moreover, hypoglycemia events were demonstrated by clinical trials regarding lixisenatide (incidence: lixisenatide, $15.3 \%$ vs. placebo, $12.3 \%$ ) (16) and albiglutide (incidence: albiglutide, $21.0 \%$ vs. placebo, $11.3 \%$ ) (17). Furthermore, a recent systematic review that enrolled 34 trials involving 14,464 patients found that all of the included GLP-1RAs (except albiglutide) were correlated with a higher risk of hypoglycemia than placebo, in which the hypoglycemia occurrence rates of different regimens ranges from $10.4 \%$ to $19.4 \%$ (3). Nevertheless, no difference of hypoglycemia risks among all the GLP-1RAs was revealed by the review, indicating no difference of hypoglycemia rate exists between long-acting and short-acting (3).

Although accumulating studies report the occurrence of hypoglycemia following the use of GLP-1RAs in patients with type 2 diabetes, most evidence comes from clinical trials $(18,19)$, which is insufficient to systematically understand the risk or characteristics of such a relatively rare adverse event. Currently, limited pharmacovigilance studies about GLP-1RAs have mainly focused on other related adverse events $(20,21)$, and little is known about the explicit features of hypoglycemia following the application of different GLP-1RAs in real-world practice. Compared to other published reports, our study is the first research focused on comparing the associations and detailed clinical features of hypoglycemia following the use of different GLP-1RAs based on a real-world pharmacovigilance database by several data mining methods.

To identify novel signals for specific adverse events from accumulating spontaneous reports collected by pharmacovigilance systems, data mining methods have been widely used, including disproportionality assessment and Bayesian analysis $(22,23)$. All disproportionality assessments are calculated based on a two-by-two contingency table, and their statistical results explain the extent to which a specific adverse event of interest is related to the suspected drug in comparison with all the other drugs included in the database. The disproportionality methodologies using proportional reporting ratio (PRR) and reporting odds ratio (ROR) were employed in our study $(24,25)$. In other approaches, Bayesian statistics were applied to overcoming variability caused by the small count in a cell of the twoby-two table (26). Bayesian confidence propagation neural network (BCPNN) and multi-item gamma Poisson shrinker (MGPS) are two representative algorithms based on Bayesian $\operatorname{logics}(23,26)$.

With these data mining methods, we aimed to compare and evaluate the associations between various GLP-1RAs and hypoglycemia by analyzing reports in the FDA's Adverse Event Reporting System (FAERS). Furthermore, we summarized onset time, fatality proportion, and hospitalization proportion for hypoglycemia following the administration 


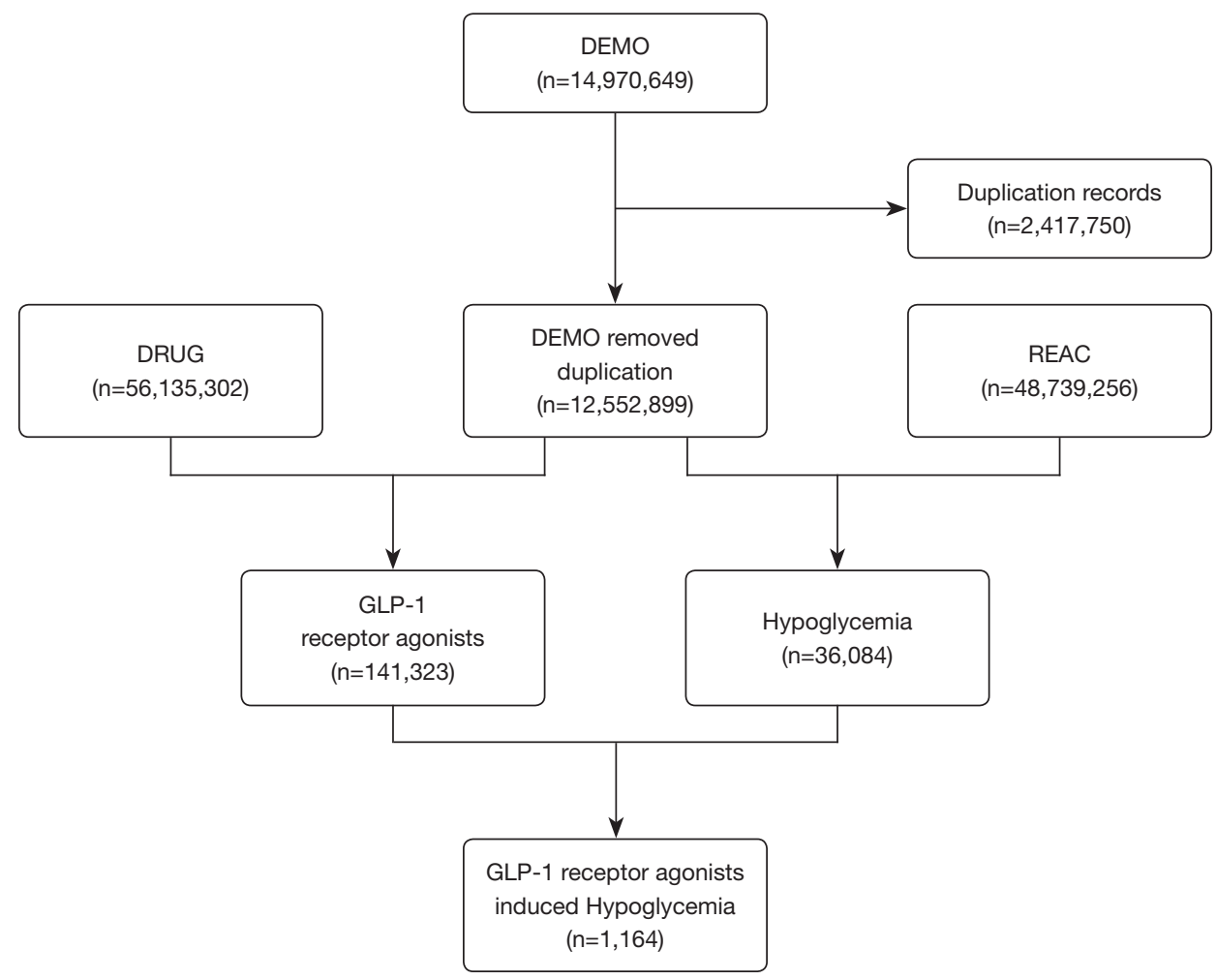

Figure 1 Selection process of cases of GLP-1RA-associated hypoglycemia from the FAERS database. DEMO, demographic and administrative information; DRUG, drug information; FAERS, Food and Drug Administration's Adverse Event Reporting System; GLP1RA, glucagon-like peptide-1 receptor agonist; REAC, adverse event.

of different GLP-1RAs. We present the following article in accordance with the STROBE reporting checklist (available at https://dx.doi.org/10.21037/atm-21-4162).

\section{Methods}

\section{Data collection}

The FAERS is a public case reporting database, which contains medication errors and adverse reactions submitted by healthcare professionals, manufactures, patients, and consumers. FAERS data files provide drug information (DRUG), demographic and administrative information (DEMO), preferred terms (PTs) coded for the adverse event (REAC), report sources (RPSR), patient outcomes (OUTC), therapy start and end dates for reported drugs (THER), and indications for use (INDI). We performed a retrospective pharmacovigilance study based on reports from the FAERS database collected between January 2004 and September 2020. A total of $12,552,899$ cases were finally selected from the FAERS database, after the removal of deduplicates (following the FDA's recommendations by choosing the latest FDA_DT when the CASEIDs were the same, as well as selecting the higher PRIMARYID when the CASEID and FDA_DT were the same) (Figure 1). The study was conducted in accordance with the Declaration of Helsinki (as revised in 2013).

\section{Data mapping}

In the REAC files, the FAERS database defines adverse events using PTs from the Medical Dictionary for Regulatory Activities (MedDRA v23.1). The PTs related to hypoglycemia were as follows: Hypoglycemia (code: 10021005), Hypoglycaemia (code: 10020993), and Hypoglycaemic conditions NEC (code: 10021001). The drugs were identified in FAERS by both generic and brand names utilizing the MICROMEDEX ${ }^{\circledR}$ (Index Nominum) as a dictionary in data mining (Table 1). We selected the agents reported as "PS" (Primary Suspect). 
Table 1 Summary of FDA-approved GLP-1RAs

\begin{tabular}{lll}
\hline Generic name & Brand name & Approval year \\
\hline Exenatide & $\begin{array}{l}\text { Bydureon, Bydureon Bcise, } \\
\text { Byetta }\end{array}$ & 2005 \\
Liraglutide & Saxenda, Victoza & 2009 \\
Albiglutide & Eperzan, Tanzeum & 2014 \\
Dulaglutide & Trulicity & 2014 \\
Lixisenatide & Adlyxin, Lyxumia & 2013 \\
Semaglutide & Ozempic, Rybelsus & 2017
\end{tabular}

FDA, Food and Drug Administration; GLP-1RAs, glucagon-like peptide-1 receptor agonists.

Table 2 Summary of major algorithms used for signal detection

\begin{tabular}{|c|c|c|}
\hline Algorithms & Equation* & Criteria \\
\hline ROR & $\begin{array}{l}\mathrm{ROR}=(\mathrm{a} / \mathrm{b}) /(\mathrm{c} / \mathrm{d}) \\
95 \% \mathrm{Cl}=\mathrm{e}^{\ln (\mathrm{ROR}) \pm 1.96(1 / \mathrm{a}+1 / \mathrm{b}+1 / \mathrm{c}+1 / \mathrm{d})^{\wedge} 0.5}\end{array}$ & $\begin{array}{l}95 \% \mathrm{Cl}>1 \\
\mathrm{~N} \geq 2\end{array}$ \\
\hline PRR & $\begin{array}{l}\operatorname{PRR}=(a /(a+c)) /(b /(b+d)) \\
\chi^{2}=\Sigma((O-E) 2 / E) ;(O=a, E=(a+b)(a+c) / \\
(a+b+c+d))\end{array}$ & $\begin{array}{l}\mathrm{PRR} \geq 2 \\
\chi^{2} \geq 4, \mathrm{~N} \geq 3\end{array}$ \\
\hline BCPNN & $\begin{array}{l}I C=\log _{2} a(a+b+c+d) /((a+c)(a+b)) \\
I C 025=e^{\ln (I C)-1.96(1 / a+1 / b+1 / c+1 / 1 / d) \wedge 0.5}\end{array}$ & IC025>0 \\
\hline MGPS & $\begin{array}{l}E B G M=a(a+b+c+d) /((a+c)(a+b)) \\
E B G M 05=e^{\ln (E B G M)-1.64(1 / a+1 / b+1 / c+1 / d)^{\wedge} 0.5}\end{array}$ & $\begin{array}{l}\text { EBGM05>2, } \\
\mathrm{N}>0\end{array}$ \\
\hline
\end{tabular}

*a, number of reports containing both the suspect drug and the suspect adverse drug reaction; $b$, number of reports containing the suspect adverse drug reaction with other medications (except the drug of interest); c, number of reports containing the suspect drug with other adverse drug reactions (except the event of interest); $d$, number of reports containing other medications and other adverse drug reactions. ROR, reporting odds ratio; $\mathrm{Cl}$, confidence interval; $\mathrm{N}$, the number of cooccurrences; PRR, proportional reporting ratio; $\chi^{2}$, chi-squared; BCPNN, Bayesian confidence propagation neural network; IC, information component; IC025, the lower limit of the 95\% twosided $\mathrm{Cl}$ of the IC; MGPS, multi-item gamma Poisson shrinker; EBGM, empirical Bayesian geometric mean; EBGM05, the lower $90 \%$ one-sided $\mathrm{Cl}$ of EBGM.

\section{Data mining}

We employed the PRR, ROR, BCPNN, and MGPS algorithms based on disproportionality and Bayesian analyses to investigate the association between an adverse event and a drug. The equations and criteria of the four algorithms are provided in Table 2. The association between different GLP-1RAs and hypoglycemia were compared. In this study, the specific GLP-1RA was the "PS" in the ROLE_COD field of DRUG files, with no other GLP1RAs being listed as the "concomitant", "second suspect", or "interacting" drug in the same report. We also assessed the onset time of hypoglycemia for different GLP-1RAs, which was defined as the interval between the onset date of an adverse event (EVENT_DT) and the start date of GLP1RA treatment (START_DT). Records with input errors (START_DT later than EVENT_DT) or inaccurate date entries were not included. In addition, reports with outcome data were filtered for further analysis, and the fatality proportion was calculated by dividing the fatal events by the total reports of GLP-1RA-related hypoglycemia.

\section{Statistical analysis}

Descriptive analysis was utilized to summarize the clinical features of patients with GLP-1RA-associated hypoglycemia retrieved from the FAERS database. The time to onset of GLP-1RA-associated hypoglycemia among different GLP-1RAs were compared using non-parametric tests (Mann-Whitney test for two groups; Kruskal-Wallis test for multiple groups) since the data did not follow a normal distribution. Fisher's exact test was used to compare the outcome events among different GLP-1RAs. A P value $<0.05$ with $95 \%$ confidence intervals was defined as statistically significant. All statistical analyses including data mining were conducted by using SAS, version 9.4 (SAS Institute Inc., Cary, NC, USA).

\section{Results}

\section{Descriptive analysis}

In total, 141,323 GLP-1RA-associated adverse events and 36,084 reported cases of hypoglycemia were included in the FAERS database from January 2004 to September 2020. Of these, 1,164 reports with suspected GLP-1RA-related hypoglycemia were identified and their clinical features are shown in Table 3. Among all adverse events, the affected patients were mainly aged 45-64 years old (36.68\%), and females $(59.45 \%)$ accounted for a larger proportion than males $(36.43 \%)$. These reports were predominantly from North America (76.89\%), followed by Europe (10.40\%) and Asia (8.33\%). Additionally, $62.11 \%$ of the reports were submitted by consumers, compared to $33.08 \%$ reported by health-professionals. The reported cases of GLP-1RA- 
Table 3 Clinical characteristics of patients with GLP-1RA-related hypoglycemia sourced from the FAERS database (from January 2004 to September 2020)

\begin{tabular}{|c|c|}
\hline Characteristics & Reports, No. (\%) \\
\hline \multicolumn{2}{|l|}{ Age } \\
\hline$<18$ & $1(0.09)$ \\
\hline $18-44$ & $72(6.19)$ \\
\hline $45-64$ & 427 (36.68) \\
\hline $65-74$ & 214 (18.38) \\
\hline $75-84$ & $69(5.93)$ \\
\hline$>84$ & $14(1.20)$ \\
\hline Unknown & $367(31.53)$ \\
\hline \multicolumn{2}{|l|}{ Gender } \\
\hline Female & $692(59.45)$ \\
\hline Male & $424(36.43)$ \\
\hline Unknown & $48(4.12)$ \\
\hline \multicolumn{2}{|l|}{ Area } \\
\hline North America & 895 (76.89) \\
\hline Europe & $121(10.40)$ \\
\hline Asia & $97(8.33)$ \\
\hline South America & $43(3.69)$ \\
\hline Oceania & $8(0.69)$ \\
\hline \multicolumn{2}{|l|}{ Reporter } \\
\hline Consumer & $723(62.11)$ \\
\hline Physician & $254(21.82)$ \\
\hline Pharmacist & $52(4.47)$ \\
\hline Other health-professional & 79 (6.79) \\
\hline Unknown & $56(4.81)$ \\
\hline \multicolumn{2}{|l|}{ Drugs } \\
\hline Exenatide & $723(62.11)$ \\
\hline Liraglutide & $178(15.29)$ \\
\hline Albiglutide & $21(1.80)$ \\
\hline Dulaglutide & $207(17.78)$ \\
\hline Lixisenatide & $13(1.12)$ \\
\hline Semaglutide & $22(1.89)$ \\
\hline
\end{tabular}

Table 3 (continued)
Table 3 (continued)

\begin{tabular}{lc}
\hline Characteristics & Reports, No. (\%) \\
\hline Indications & $10(0.86)$ \\
Type 1 diabetes mellitus & $534(45.88)$ \\
Type 2 diabetes mellitus & $334(28.69)$ \\
Diabetes mellitus-unspecified & $18(1.55)$ \\
Abnormal blood glucose & $19(1.63)$ \\
Weight control & $249(21.39)$ \\
Unknown &
\end{tabular}

associated hypoglycemia ascended fluctuantly from 2005 to 2020 (Figure 2). The analysis revealed that exenatide was the PS (62.11\%) in most reports. Hypoglycemia adverse events were most frequently reported in patients with Type 2 Diabetes Mellitus ( $\mathrm{n}=534,45.88 \%$ ), followed by patients with unspecified diabetes mellitus ( $\mathrm{n}=334,28.69 \%)$.

\section{Disproportionality and Bayesian analyses}

We detected the signals of hypoglycemia adverse events for all six GLP-1RA agents by utilizing four algorithms and the corresponding criteria. As shown in Table 4, lixisenatide exhibited the most noteworthy association with hypoglycemia adverse effects, owing to its highest ROR, PRR, and empirical Bayesian geometric mean (EBGM), followed by exenatide, dulaglutide, liraglutide, and semaglutide. Meanwhile, no relationship between albiglutide and hypoglycemia was observed, as its ROR, PRR, and EBGM signals did not meet the criteria.

\section{Onset time of GLP-1RA-related bypoglycemia}

In general, the median time to onset of GLP-1RA-related hypoglycemia was 5 days [interquartile range (IQR), 0-67.75 days]. The time to onset of hypoglycemia adverse event following each GLP-1RA regimen are exhibited in Figure 3. Notably, hypoglycemia was found to occur as early as the first usage in all GLP-1RAs, according to the identical dates of drug treatment and GLP-1RA-associated hypoglycemia onset time. The median onset time to events 
Reporting cases and proportions in different years

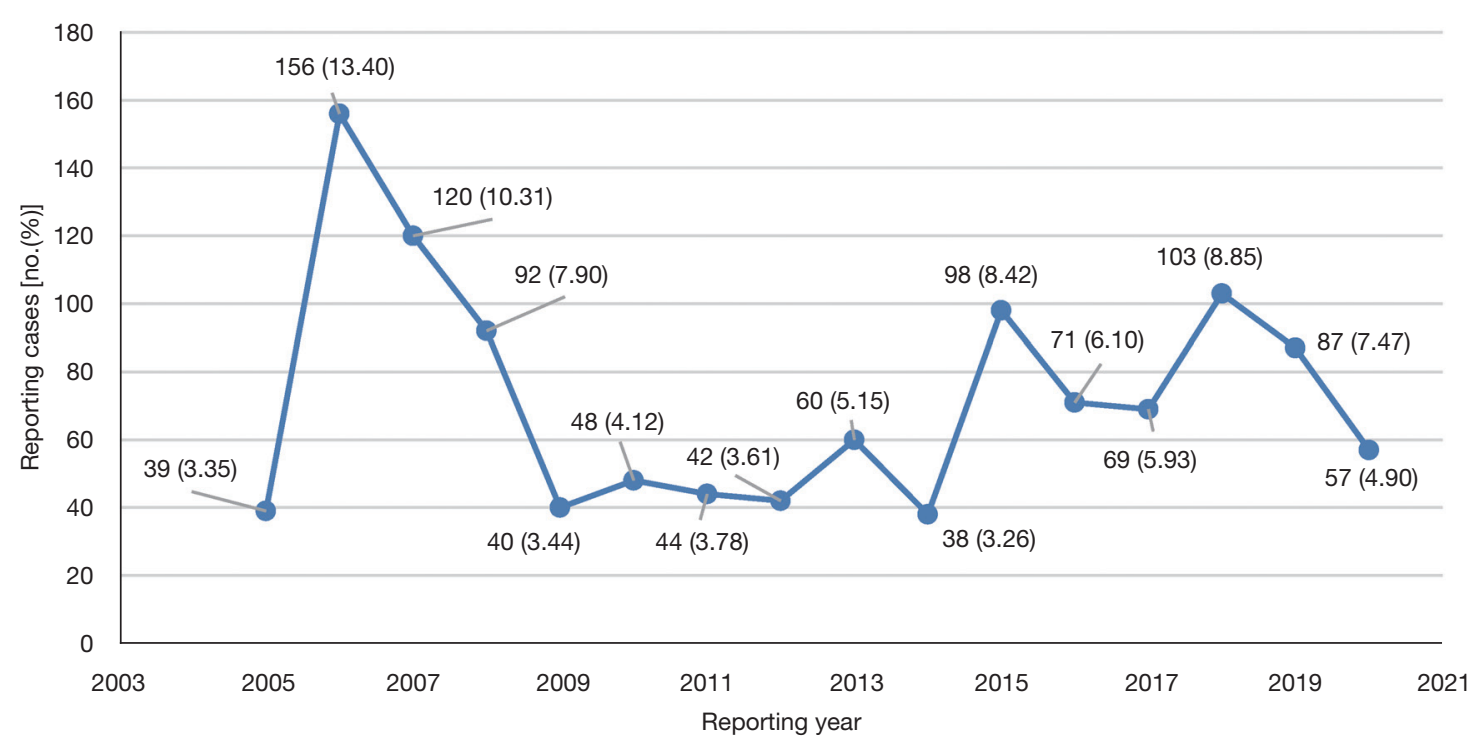

Figure 2 Numbers and proportions of hypoglycemia cases after receiving GLP-1RAs in different years. GLP-1RA, glucagon-like peptide-1 receptor agonist.

Table 4 Association of different GLP-1RAs with hypoglycemia

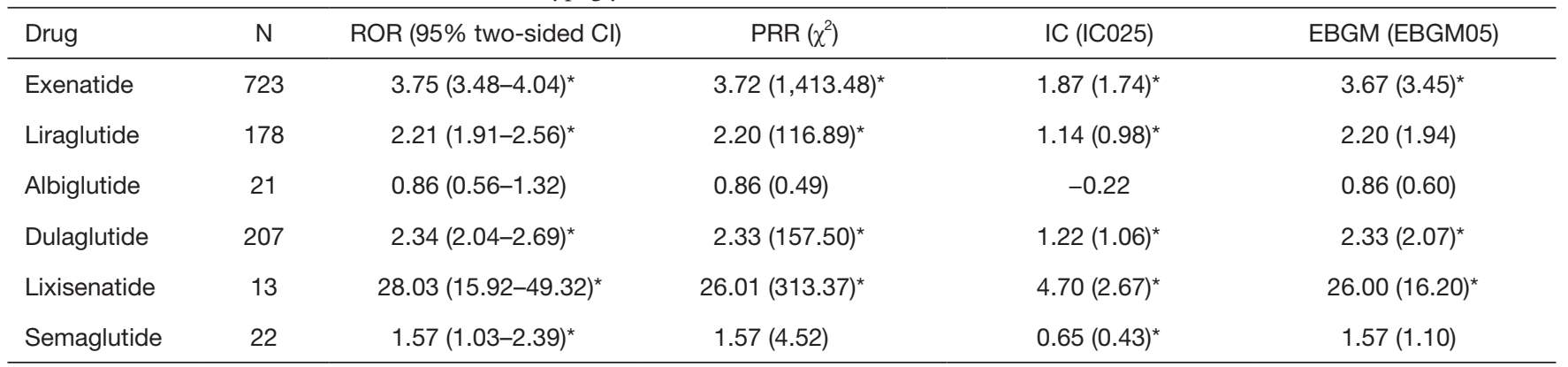

*, signals. GLP-1RA, glucagon-like peptide-1 receptor agonist; N, the number of reports of GLP-1RA associated hypoglycemia; ROR, reporting odds ratio; $\mathrm{Cl}$, confidence interval; PRR, proportional reporting ratio; $\chi^{2}$, chi-squared; IC, information component; EBGM, empirical Bayes geometric mean.

among various GLP-1RAs were as follows: 5 days (IQR, 0-73.25 days) for exenatide; 17 days (IQR, 1-95.75 days) for liraglutide; 2 days (IQR, 1-6 days) for albiglutide; 2 days (IQR, 0-22.5 days for dulaglutide; and 2 days (IQR, 0.7565.75 days) for semaglutide. In addition, $70.04 \%, 70.45 \%$, $100.00 \%, 83.72 \%, 90.00 \%$, and $62.50 \%$ of the adverse events occurred within 60 days for exenatide, liraglutide, albiglutide, dulaglutide, lixisenatide, and semaglutide, respectively. No significant differences in the onset time of hypoglycemia among these GLP-1RAs were detected (Kruskal-Wallis test, $\mathrm{P}=0.2799$ ).

\section{Outcomes of GLP-1RA-related bypoglycemia}

We assessed the proportions of hospitalization and fatality resulting from hypoglycemia adverse events after various GLP-1RA administrations to determine the prognosis of GLP-1RA-related hypoglycemia. Only patients with outcome data were included in this analysis. As shown in Figure 4, hypoglycemia generally portended poor outcomes, with fatality and hospitalization proportions of $3.53 \%$ and $56.08 \%$, respectively. Overall, the highest fatality proportion occurred following the use of liraglutide (8.33\%), 


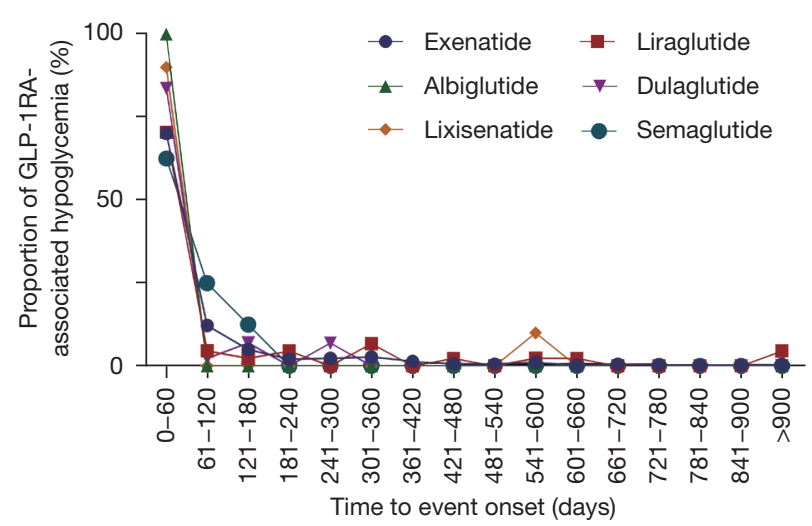

Figure 3 Time to event onset of hypoglycemia following different GLP1-RA regimens. GLP-1RA, glucagon-like peptide-1 receptor agonist.

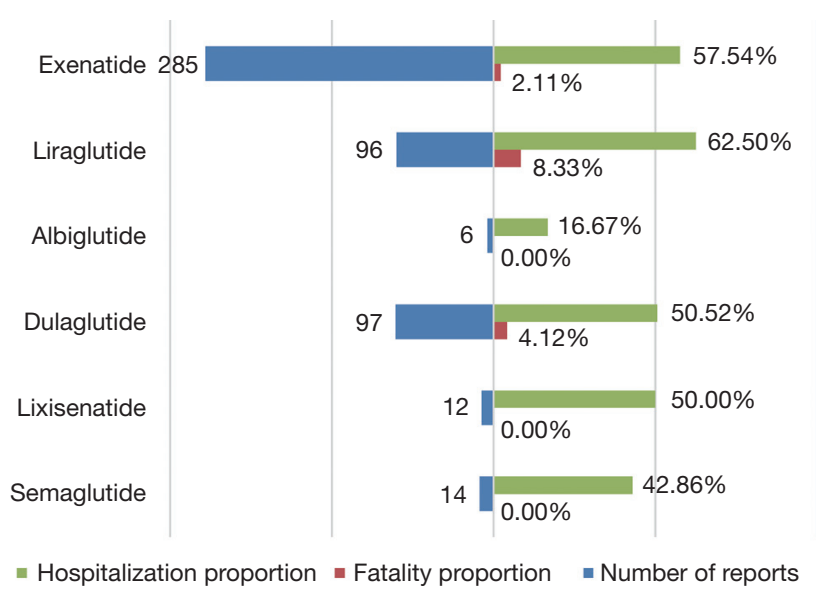

Figure 4 The number of total reports, hospitalization proportions, and fatality proportions of GLP-1RA-associated hypoglycemia. GLP-1RA, glucagon-like peptide-1 receptor agonist.

and dulaglutide (4.12\%) ranked second. Nevertheless, no significant differences existed in the fatality proportions of all GLP-1RA treatments based on the sourced data. In our analysis, liraglutide was found to have the highest hospitalization proportion $(62.50 \%)$, followed by exenatide $(57.54 \%)$, dulaglutide $(50.52 \%)$, lixisenatide $(50.00 \%)$, and semaglutide $(42.86 \%)$, while albiglutide-induced hypoglycemia contributed to the lowest hospitalization proportion (16.67\%). Additionally, the hospitalization proportion following the use of liraglutide was statistically higher than that of albiglutide $(62.50 \%$ vs. $16.67 \%$, $\mathrm{P}=0.0373)$.

\section{Discussion}

To our knowledge, this research is the first and largest study conducted thus far comparing the associations, onset, and outcomes of hypoglycemia following the use of different GLP-1RAs in real-world data based on analyzing the FAERS pharmacovigilance database. All GLP-1RA regimens (except albiglutide) demonstrated a strong association with hypoglycemia. Also, these GLP-1RAs were exhibited with diverse characteristics.

Exenatide was the first GLP-1RA approved by the FDA to treat type 2 diabetes in 2005, and has greatly improved glucose control. In an early clinical trial, exenatide combined with sulphonylureas resulted in an elevated incidence of mild-to-moderate hypoglycemia (27). Furthermore, a Korean post-marketing study revealed that hypoglycemia occurred in $1.72 \%$ of enrolled patients treated with exenatide in addition to original anti-diabetic therapies (28). Moreover, hypoglycemia was reported as an adverse event related to liraglutide both in primary clinical trials and post-marketing surveillance $(15,29,30)$. In addition, hypoglycemia was also present in patients treated with other GLP-1RAs, like lixisenatide (16), albiglutide (17), dulaglutide (31), and semaglutide (32). However, these investigations lacked detailed characterization of GLP1RA-associated hypoglycemia, owing to its low incidence. Recently, an increasing number of clinical trials have reported cases of GLP-1RA-associated hypoglycemia. Although clinical trials can provide powerful evidence to evaluate the efficacy of novel drugs, their ability to draw definitive conclusions about drug safety is limited due to the finite scope, restricted time frame, strict study entry criteria, and relatively small sample sizes. Nevertheless, spontaneous reporting systems (SRSs) can be used as a primary source of post-marketing data, which is important for the detection of safety issues, including GLP-1RA-related hypoglycemia. With the rapid progress of research on GLP-1RAs, an increasing number of patients will receive these novel regimens for plasma glucose control. Hence, it is important to determine the relationship between particular GLP1RAs and hypoglycemia as well as the detailed clinical manifestations, and to raise awareness about the possibility of this adverse event among physicians.

According to our descriptive results based on the FAERS database, our results suggested that GLP-1RAassociated hypoglycemia was mainly detected in younger patients $(42.96 \%<65$ years vs. $25.52 \% \geq 65$ years $)$, which 
might be due to the fact that the majority of diabetes patients were aged 45-64 years (33). However, this finding cannot indicate how age affects the occurrence of hypoglycemia in diabetes patients receiving GLP-1RAs. Therefore, further investigation is necessary to determine the relationship between age and incidence of GLP1RA-induced hypoglycemia. In addition, GLP-1RAassociated hypoglycemia seemed to predominately affect females $(59.45 \%)$, which is consistent with the finding that hypoglycemia in type 2 diabetes occurred more often in females $(53.20 \%$ ) (34). However, more investigations are still needed to elucidate the influence of gender on the occurrence of GLP-1RA-related hypoglycemia. Moreover, a fluctuant increase in reported cases over time was observed, which may have resulted from the elevated numbers of approved drugs and optimized administration of GLP-1RAs in clinical practice. Notably, 19 hypoglycemia patients were administered GLP-1RAs for weight control, which were off-label usages of these drugs. In these cases, it would be quite dangerous that exercising might also be utilized for weight control. Therefore, we should strictly follow the drug use standards to avoid these unwanted, severe side effects. Taken together, our current research describes some basic characteristics of patients suffering from GLP-1RAassociated hypoglycemia.

In the pharmacovigilance investigation, we found all six GLP-1RAs (except albiglutide) were associated with hypoglycemia. Interestingly, a meta-analysis comparing the safety profiles from 34 clinical trials of GLP-1RAs demonstrated that all the regimens except albiglutide elevated the risk of hypoglycemia, which is consistent with our findings (3). It is important to note that lixisenatide exhibited the strongest relationship to hypoglycemia among all of the drugs, while exenatide demonstrated a relevantly weaker association. In a study analyzing the risk of hypoglycemia after glucose-lowering treatment utilizing the Japanese Adverse Drug Event Report (JADER) database, lixisenatide also showed higher ROR and IC values than the other two GLP-1RAs, exenatide and liraglutide (35). However, a clinical trial revealed that patients who received lixisenatide showed a lower incidence of hypoglycemia than patients receiving twice-daily exenatide (lixisenatide, $2.5 \%$ vs. twice-daily exenatide, $7.9 \% ; \mathrm{P}<0.05)(16)$. Although our study provides signals for hypoglycemia following the use of GLP-RAs, it is difficult to explain the results of different reports due to the limited evidence available presently. Therefore, more investigations like experimental exploration, clinical trials, case-control studies, and cohort studies are needed to validate our findings. Yet, we suggest that physicians should pay more attention to patients with diabetes who take GLP1-RAs for glucose control. For these patients, regular monitoring of glucose is recommended.

In addition, we found that the median onset time of hypoglycemia was 5 days (IQR 0-67.75) following the administration of GLP-1RAs. In general, the risk of hypoglycemia decreased over time, and these GLP-1RAs resulted in a hypoglycemia rate of $62.50-100.00 \%$ at 2 months after treatment in the real-world analysis. Moreover, statistical analysis found no significant difference in the onset time of hypoglycemia among these GLP-1RA drugs. It seems that albiglutide, dulaglutide, and semaglutide induce hypoglycemia in the extremely short median onset time of 2 days, while hypoglycemia may strike patients who receive exenatide or liraglutide even after 2 years. The onset time of GLP-1RA-related hypoglycemia needs to be further investigated. The huge distinctions in the onset time among different GLP-1RA regimens suggest that individualized monitoring methods should be performed after treatment. Moreover, careful monitoring in the early period following GLP-1RA administration may detect most hypoglycemia events.

To further assess the severity of hypoglycemia related to different GLP-1RAs, the fatality and hospitalization proportions caused by hypoglycemia following the use of different GLP-1RA drugs were compared. Generally, GLP1RAs led to fatality and hospitalization proportions of $3.53 \%$ and $56.08 \%$, respectively. Despite the low general fatality proportion, liraglutide $(8.33 \%)$ and dulaglutide $(4.12 \%)$ treatments could induce relatively high fatality proportions. In contrast, the death risk of hypoglycemia related to exenatide was lower, indicating that hypoglycemia in patients receiving exenatide is less severe. No death reports were witnessed in the remaining three groups, possibly due to the small numbers of hypoglycemia cases. Surprisingly, the hospitalization proportions of hypoglycemia cases associated with GLP-1RAs were all significantly higher than the fatality proportions, indicating the majority of the diabetes patients present with hypoglycemia after GLP-RA treatments may have severe symptoms and receive intensive care after the occurrence of this adverse event. Despite the statistically higher hospitalization proportion in liraglutide than albiglutide, the accuracy of albiglutide-associated hypoglycemia data might be limited by its low reporting number. Therefore, it is necessary to include more reports in the future to confirm our results. Collectively, these data suggest that GLP-1RA-related hypoglycemia is severe, 
despite the low risk of death.

With increasing evidence confirming the valid efficacy of GLP-1RAs in various applications, clinicians must be attentive to hypoglycemia in different agents. The present study can be used to inform clinical decisions on choosing GLP-RAs, identify patients with high possibility of developing hypoglycemia, and organize individualized monitoring. The onset time analysis of GLP-1RA-related hypoglycemia in our research suggests that regular and careful monitoring of plasma glucose can identify most hypoglycemia events in the early period of treatment. During this period, hypoglycemia symptoms should also be paid attention to, like tremor, palpitations, sweating, headache, blurred vision, dizziness, and nausea, etc. (36). Once hypoglycemia is detected in the patients, dose adjustment of anti-diabetic drugs and hourly plasma glucose monitoring should be immediately adopted to avoid the occurrence of severe hypoglycemia (36). According to a case of hypoglycemia associated with liraglutide use, supportive measures can also be taken to alleviate symptoms (37). Moreover, a recent case report about hypoglycemia showed that a female diabetes patient had not recovered from liraglutide-related hypoglycemia until she received dextrose infusion, which suggests that dextrose supplementation is important if hypoglycemia persists (38).

Despite of the advantages of the data mining strategies utilized in our analysis and the real-world research based on the FAERS database, it should be noted that some particular limitations of SRS-based research are unavoidable. Firstly, some inherent limitations of SRS itself, such as false reporting, underreporting, inaccurate reporting, and incomplete reporting, can hardly be solved by data mining techniques, which may lead to inevitable bias. Secondly, only cases with adverse events are covered in SRS data. Since the total numbers of cases undergoing treatment with different GLP-1RAs are unknown, the incidence of hypoglycemia for each drug cannot be calculated and compared. Thirdly, it is challenging to identify the exact risk factors of hypoglycemia in patients receiving GLP-1RA without baseline plasma glucose levels, glycated hemoglobin, and C-peptide information. Fourthly, the association signals demonstrated in the current study can hardly prove a causal relationship. However, despite the unavoidable limitations that exist in our research, it can still profile some vital aspects of GLP-1RA-related hypoglycemia, and provide clues for further prospective clinical studies regarding this association.

\section{Conclusions}

In this study, our investigation based on the real-world FAERS database identifies signals for hypoglycemia related to different GLP-1RAs. The most significant finding of our current research is that lixisenatide shows the strongest association with hypoglycemia among all six GLP-RA drugs. Although further pharmacoepidemiological investigations are needed to verify our hypotheses, our results are critical for additional surveillance and investigation in this area.

\section{Acknowledgments}

Funding: None.

\section{Footnote}

Reporting Checklist: The authors have completed the STROBE reporting checklist. Available at https://dx.doi. org/10.21037/atm-21-4162

Conflicts of Interest: All authors have completed the ICMJE uniform disclosure form (available at https://dx.doi. org/10.21037/atm-21-4162). The authors have no conflicts of interest to declare.

Ethical Statement: The authors are accountable for all aspects of the work in ensuring that questions related to the accuracy or integrity of any part of the work are appropriately investigated and resolved. The study was conducted in accordance with the Declaration of Helsinki (as revised in 2013).

Open Access Statement: This is an Open Access article distributed in accordance with the Creative Commons Attribution-NonCommercial-NoDerivs 4.0 International License (CC BY-NC-ND 4.0), which permits the noncommercial replication and distribution of the article with the strict proviso that no changes or edits are made and the original work is properly cited (including links to both the formal publication through the relevant DOI and the license). See: https://creativecommons.org/licenses/by-nc-nd/4.0/.

\section{References}

1. Meier JJ. GLP-1 receptor agonists for individualized treatment of type 2 diabetes mellitus. Nat Rev Endocrinol 2012;8:728-42. 
2. Eng C, Kramer CK, Zinman B, et al. Glucagonlike peptide-1 receptor agonist and basal insulin combination treatment for the management of type 2 diabetes: a systematic review and meta-analysis. Lancet 2014;384:2228-34.

3. Htike ZZ, Zaccardi F, Papamargaritis D, et al. Efficacy and safety of glucagon-like peptide-1 receptor agonists in type 2 diabetes: A systematic review and mixed-treatment comparison analysis. Diabetes Obes Metab 2017;19:524-36.

4. Marso SP, Daniels GH, Brown-Frandsen K, et al. Liraglutide and Cardiovascular Outcomes in Type 2 Diabetes. N Engl J Med 2016;375:311-22.

5. Marso SP, Bain SC, Consoli A, et al. Semaglutide and Cardiovascular Outcomes in Patients with Type 2 Diabetes. N Engl J Med 2016;375:1834-44.

6. Hernandez AF, Green JB, Janmohamed S, et al. Albiglutide and cardiovascular outcomes in patients with type 2 diabetes and cardiovascular disease (Harmony Outcomes): a double-blind, randomised placebo-controlled trial. Lancet 2018;392:1519-29.

7. Gerstein HC, Colhoun HM, Dagenais GR, et al. Dulaglutide and cardiovascular outcomes in type 2 diabetes (REWIND): a double-blind, randomised placebocontrolled trial. Lancet 2019;394:121-30.

8. Buse JB, Wexler DJ, Tsapas A, et al. 2019 Update to: Management of Hyperglycemia in Type 2 Diabetes, 2018. A Consensus Report by the American Diabetes Association (ADA) and the European Association for the Study of Diabetes (EASD). Diabetes Care 2020;43:487-93.

9. Davies MJ, D'Alessio DA, Fradkin J, et al. Management of Hyperglycemia in Type 2 Diabetes, 2018. A Consensus Report by the American Diabetes Association (ADA) and the European Association for the Study of Diabetes (EASD). Diabetes Care 2018;41:2669-701.

10. Filippatos TD, Panagiotopoulou TV, Elisaf MS. Adverse Effects of GLP-1 Receptor Agonists. Rev Diabet Stud 2014;11:202-30.

11. Sun F, Chai S, Yu K, et al. Gastrointestinal adverse events of glucagon-like peptide-1 receptor agonists in patients with type 2 diabetes: a systematic review and network meta-analysis. Diabetes Technol Ther 2015;17:35-42.

12. Singh S, Chang HY, Richards TM, et al. Glucagonlike peptide 1-based therapies and risk of hospitalization for acute pancreatitis in type 2 diabetes mellitus: a populationbased matched case-control study. JAMA Intern Med 2013;173:534-9.

13. Nauck $M$. Incretin therapies: highlighting common features and differences in the modes of action of glucagon-like peptide-1 receptor agonists and dipeptidyl peptidase-4 inhibitors. Diabetes Obes Metab 2016;18:203-16.

14. Shyangdan DS, Royle P, Clar C, et al. Glucagon-like peptide analogues for type 2 diabetes mellitus. Cochrane Database Syst Rev 2011;(10):CD006423.

15. Marre $M$, Shaw J, Brändle $M$, et al. Liraglutide, a oncedaily human GLP-1 analogue, added to a sulphonylurea over 26 weeks produces greater improvements in glycaemic and weight control compared with adding rosiglitazone or placebo in subjects with Type 2 diabetes (LEAD-1 SU). Diabet Med 2009;26:268-78.

16. Rosenstock J, Hanefeld M, Shamanna P, et al. Beneficial effects of once-daily lixisenatide on overall and postprandial glycemic levels without significant excess of hypoglycemia in type 2 diabetes inadequately controlled on a sulfonylurea with or without metformin (GetGoal-S). J Diabetes Complications 2014;28:386-92.

17. Home PD, Shamanna P, Stewart M, et al. Efficacy and tolerability of albiglutide versus placebo or pioglitazone over 1 year in people with type 2 diabetes currently taking metformin and glimepiride: HARMONY 5. Diabetes Obes Metab 2015;17:179-87.

18. Pencek R, Brunell SC, Li Y, et al. Exenatide once weekly for the treatment of type 2 diabetes mellitus: clinical results in subgroups of patients using different concomitant medications. Postgrad Med 2012;124:33-40.

19. Seino Y, Min KW, Niemoeller E, et al. Randomized, double-blind, placebo-controlled trial of the once-daily GLP-1 receptor agonist lixisenatide in Asian patients with type 2 diabetes insufficiently controlled on basal insulin with or without a sulfonylurea (GetGoal-L-Asia). Diabetes Obes Metab 2012;14:910-7.

20. Pradhan R, Montastruc F, Rousseau V, et al. Exendinbased glucagon-like peptide-1 receptor agonists and anaphylactic reactions: a pharmacovigilance analysis. Lancet Diabetes Endocrinol 2020;8:13-4.

21. Mali G, Ahuja V, Dubey K. Glucagon-like peptide-1 analogues and thyroid cancer: An analysis of cases reported in the European pharmacovigilance database. J Clin Pharm Ther 2021;46:99-105.

22. Kubota K, Koide D, Hirai T. Comparison of data mining methodologies using Japanese spontaneous reports. Pharmacoepidemiol Drug Saf 2004;13:387-94.

23. Sakaeda T, Tamon A, Kadoyama K, et al. Data mining of the public version of the FDA Adverse Event Reporting System. Int J Med Sci 2013;10:796-803.

24. Montastruc JL, Sommet A, Bagheri H, et al. Benefits 
and strengths of the disproportionality analysis for identification of adverse drug reactions in a pharmacovigilance database. Br J Clin Pharmacol 2011;72:905-8.

25. Ooba N, Kubota K. Selected control events and reporting odds ratio in signal detection methodology. Pharmacoepidemiol Drug Saf 2010;19:1159-65.

26. Bate A. Bayesian confidence propagation neural network. Drug Saf 2007;30:623-5.

27. Buse JB, Henry RR, Han J, et al. Effects of exenatide (exendin-4) on glycemic control over 30 weeks in sulfonylurea-treated patients with type 2 diabetes. Diabetes Care 2004;27:2628-35.

28. Hwang YC, Kim A, Jo E, et al. Effectiveness and safety of exenatide in Korean patients with type 2 diabetes inadequately controlled with oral hypoglycemic agents: an observational study in a real clinical practice. BMC Endocr Disord 2017;17:68.

29. Martinez L, Penfornis A, Gautier JF, et al. Effectiveness and Persistence of Liraglutide Treatment Among Patients with Type 2 Diabetes Treated in Primary Care and Specialist Settings: A Subgroup Analysis from the EVIDENCE Study, a Prospective, 2-Year Followup, Observational, Post-Marketing Study. Adv Ther 2017;34:674-85.

30. Russell-Jones D, Vaag A, Schmitz O, et al. Liraglutide vs insulin glargine and placebo in combination with metformin and sulfonylurea therapy in type 2 diabetes mellitus (LEAD-5 met+SU): a randomised controlled trial.
Diabetologia 2009;52:2046-55.

31. Zhang L, Zhang M, Zhang Y, et al. Efficacy and safety of dulaglutide in patients with type 2 diabetes: a meta-analysis and systematic review. Sci Rep 2016;6:18904.

32. Andreadis P, Karagiannis T, Malandris K, et al. Semaglutide for type 2 diabetes mellitus: A systematic review and meta-analysis. Diabetes Obes Metab 2018;20:2255-63.

33. Wild S, Roglic G, Green A, et al. Global prevalence of diabetes: estimates for the year 2000 and projections for 2030. Diabetes Care 2004;27:1047-53.

34. Chan SP, Ji LN, Nitiyanant W, et al. Hypoglycemic symptoms in patients with type 2 diabetes in Asia-PacificReal-life effectiveness and care patterns of diabetes management: the RECAP-DM study. Diabetes Res Clin Pract 2010;89:e30-2.

35. Gosho M. Risk of Hypoglycemia After Concomitant Use of Antidiabetic, Antihypertensive, and Antihyperlipidemic Medications: A Database Study. J Clin Pharmacol 2018;58:1324-31.

36. Iqbal A, Heller S. Managing hypoglycaemia. Best Pract Res Clin Endocrinol Metab 2016;30:413-30.

37. Elmehdawi RR, Elbarsha AM. An accidental liraglutide overdose: case report. Libyan J Med 2014;9:23055.

38. Solverson KJ, Lee H, Doig CJ. Intentional overdose of liraglutide in a non-diabetic patient causing severe hypoglycemia. CJEM 2018;20:S61-3.

(English Language Editor: A. Kassem)
Cite this article as: Zhao Z, Tang Y, Hu Y, Zhu H, Chen X, Zhao B. Hypoglycemia following the use of glucagon-like peptide-1 receptor agonists: a real-world analysis of postmarketing surveillance data. Ann Transl Med 2021;9(18):1482. doi: $10.21037 /$ atm-21-4162 